\title{
Avaliação de custo do sistema de medição individualizado de água considerando à tarifação mínima e diferentes cenários de consumo
}

\section{Cost evaluation of the individualized water metering system considering minimum}

\author{
SILVA, Leandro Rodrigues da'; MAGALHÃES, Murilo Ribeiro²; MARTINS, Tuane \\ Rodrigues $^{3}$, SOUZA, Saulo Bruno Silveira4; REIS, Ricardo Prado Abreu 5 \\ ${ }^{1}$ Escola de Engenharia Civil da Universidade Federal de Goiás, leandrosilvaufg@gmail.com \\ ${ }^{2}$ Escola de Engenharia Civil da Universidade Federal de Goiás, murilorm10@ hotmail.com \\ ${ }^{3}$ Escola de Engenharia Civil da Universidade Federal de Goiás, tuanerodriguesmartins@ gmail.com \\ ${ }^{4}$ Escola de Engenharia Civil da Universidade Federal de Goiás, saulobrunosouza@gmail.com \\ 5 Escola de Engenharia Civil da Universidade Federal de Goiás, ricardo_reis@ufg.br
}

\begin{abstract}
RESUMO
O presente trabalho tem como objetivo avaliar o custo proporcional da incidência de tarifação mínima no sistema de medição individualizado (SMI) analisando a estrutura tarifária adotada pela SANEAGO em Goiânia-GO. Para tanto, foram obtidos dados de consumo médio mensal ( $\mathrm{m}^{3} / \mathrm{mês}$ ) e número de agentes consumidores em unidades habitacionais de diferentes edifícios multifamiliares. Os dados obtidos foram utilizados para a criação de cenários hipotéticos de moradias com diferentes proporções de agentes consumidores a fim de comparar os cenários com SMI e com a utilização do sistema de medição coletiva (SMC). Os resultados deste estudo indicaram que, para o SMC, a maioria dos casos que passa a ser benéfico possuem economias com grande número de agentes consumidores por unidade. Considerando a estrutura tarifária da SANEAGO, nenhum dos cenários com SMC foi benéfico para unidades consumidoras com apenas um morador. Apesar disto, é importante ressaltar que a estrutura tarifária adotada pelas concessionárias interfere no que diz respeito a viabilidade econômica individual devido ao sistema de medição de água adotado.
\end{abstract}

Palavras-chave: Medição individualizada de água, tarifação, viabilidade econômica.

\begin{abstract}
This paper aims to evaluate the proportional cost of the incidence of minimum charging in the water individualized measurement system (IMS) by analyzing the tariff structure adopted by SANEAGO in Goiânia-GO. For the development of this study, data were obtained on average monthly consumption $\left(\mathrm{m}^{3}\right.$ ( month) and number of consumer agents in housing units of different multifamily buildings. The data obtained were used to create hypothetical scenarios with different proportions of consumer agents in order to compare the collective measurement system (CMS) with SMI. The results of this study indicated that, for SMC, most of the cases that become beneficial have economies with large numbers of consuming agents per unit. Considering SANEAGO's tariff structure, none of the scenarios with SMC was beneficial for consumer units with only one resident. Nevertheless, it is important to emphasize that the tariff structure adopted by the concessionaires interferes with regard to the individual economic viability due to the adopted water metering system.
\end{abstract}

Keywords: Water Measurement, Charging Structure Water Consumption, Economic Viability 


\section{INTRODUÇÃO}

O aumento da demanda de água associado ao crescimento constante da população ao longo dos anos, a verticalização das edificações e a preocupação com a escassez de água potável para consumo humano exigiu às cidades a adoção de práticas de conservação e uso racional de água. Dentre elas, a mudança em edifícios multifamiliares do Sistema de Medição Coletiva (SMC) para o Sistema de Medição Individualizada (SMI).

Guedes e Junior (2015) comentam que os SMI apresentam vantagens com relação os SMC, pois o método de medição coletiva além de ser um ponto de vista econômico injusto, não conscientiza a população sobre o uso racional de água. Por outro lado, o SMI ao ser implantado concede a cada unidade consumidora, o controle sobre a conta de água, tornando-a mais ajustada ao volume realmente consumido e incentivando uma maior conscientização sobre o uso racional de água.

O Art. $2^{\circ}$, da Lei ${ }^{\circ} 13.312$ (Brasil, 2016), das Diretrizes Nacionais para o Saneamento Básico, obriga os novos edifícios a adotar hidrômetros individuais para medir o consumo de água por apartamento, sendo que esta entra em vigência a partir de 2021. Estudos especializados como os realizados pela Agência Nacional de Águas (ANA, 2005), mostram que o SMI de água implantado em edificações residenciais tem resultado em reduções médias no consumo da ordem de $25 \%$.

Com relação ao custo cobrado pelo fornecimento de água, a estrutura tarifária é composta por uma tarifa básica e três de consumo: (1) o custo mínimo fixo do serviço de suprimento de água, (2) a tarifa de água e (3) a tarifa de coleta e tratamento de esgoto. Todas variam conforme a categoria da unidade consumidora e, os três últimos, também variam conforme o consumo mensal de água (AGR, 2018).

Apesar do melhor ajustamento do custo de consumo de água proporcionado pelos SMI, analisando a estrutura de tarifação, haverá cenários onde a proporção da tarifa mínima fixa em relação à cobrança do consumo total gerado pela unidade poderá atingir valores significativos, principalmente em economias com baixo número de agentes consumidores ou em unidades consumidoras não habitadas ou em não funcionamento. Assim, ressaltase a importância de um estudo de análise econômica da estruturação tarifária adotada pelas concessionárias com o intuído de verificar a real cobrança pela água consumida e $\mathrm{o}$ custo associado a implantação dos SMI de água.

Com base na questão de estudo levantada, o objetivo deste trabalho consiste no estudo de diferentes cenários de agentes por unidade consumidora a fim de avaliar o custo proporcional da incidência de tarifação mínima no sistema de medição individualizado (SMI) analisando a estrutura tarifária adotada pela SANEAGO em Goiânia-GO. 


\section{SISTEMA DE MEDIÇÃO DE ÁGUA}

\subsection{Sistema de medição coletiva (SMC)}

Segundo Carvalho Jr. e Silveira (2008), os edifícios construídos que utilizam o SMC definem-se pela passagem da água proveniente da rede pública por um único hidrômetro principal que vai em direção aos reservatórios da edificação. O SMC é um sistema cujo custo de implantação é relativamente barato, pois o seu sistema é de baixa complexidade e fácil medição. Uma vez medido o consumo de água geral para todo o edifício, a água é distribuída, sem a necessidade de medição (ROZAS, PRADO, 2002).

O SMC consiste na leitura de um macromedidor, localizado na interface do alimentador predial no qual fornecerá a diferença entre as aferições do mês anterior e o próximo. $\mathrm{O}$ custo associado a esta diferença é dividido igualmente entre todas as unidades consumidoras do edifício (PERES, 2006).

Segundo Carvalhães (2016), um dos problemas enfrentados por edifícios projetados com um medidor coletivo de água é a incapacidade do usuário de ter conhecimento do quanto foi consumido individualmente e a aplicação de uma iniciativa própria de economia de água. Vazamentos também contêm grandes custos mensais e a medição coletiva dificulta o procedimento de localização do vazamento por existir apenas um medidor para múltiplas residências e tubulações.

\subsection{Sistema de medição individualizada (SMI)}

O SMI do consumo de água é um sistema composto por um macromedidor geral e vários medidores menores para cada unidade consumidora. Em edifícios multifamiliares, esse sistema vem sendo implantado com objetivo de fazer uma cobrança mais justa, propiciando que, de certa forma, os moradores tomem consciência sobre a importância de se economizar água e evitar desperdícios. Segundo a ANA (2005), o SMI nada mais é que uma apuração do consumo e faturamento de água. Segundo Carvalho JR. e Silveira (2008), há uma procura "por parte dos próprios moradores de edifícios pelo sistema de medição individualizada de água”.

Segundo Chaib (2016), há duas tipologias de instalação de SMI: (1) Instalação de um hidrômetro para cada apartamento, na qual a medição fica sob responsabilidade da concessionária de água e esgoto; (2) Instalação de um hidrômetro central no edifício, cuja leitura é realizada pela concessionária de água e esgoto e, também, de hidrômetros instalados instalados em cada unidade ou apartamento, cujas leituras são de responsabilidade do condomínio.

Existem várias pesquisas e análises que apontam a considerável redução de consumo de água em edificações que optaram pela adaptação de SMI em edifícios originalmente com SMC. Tal adaptação, na maioria dos casos, promove redução de consumo de água, como apontam Peruch e Back (2013) e Yamada et. al. (2001), variando entre 15 a 30\% de economia. 


\subsection{Estrutura de tarifação}

As empresas de saneamento no Brasil adotam uma estrutura tarifária bastante semelhante, a tarifação em blocos, onde é estabelecido um custo pela água $\left(\mathrm{R} \$ / \mathrm{m}^{3}\right)$ para cada faixa de consumo, delineada por um faixa de $\mathrm{m}^{3} / \mathrm{mês}$ que varia para cada concessionária. A tarifa de esgoto varia juntamente com as faixas e consiste, usualmente de um valor referente ao custo da água, frequentemente $100 \%$, ou seja, a tarifa de consumo de água é igual a de esgoto (coleta, afastamento e tratamento).

Ao avaliar o sistema de tarifação adotado por diversas concessionarias, tanto para o SMI como para SMC, seguem o mesmo princípio. O que difere de um para outro é a tarifação mínima, a distribuição dos blocos de consumo e suas respectivas taxas.

Em Goiânia - GO, com atuação da concessionária SANEAGO, a tarifação mínima não existe. Por outro lado, há uma tarifa básica, com o suporte legal do $\$ 8$ do artigo 57 da Lei 14.939 (15 de setembro de 2004) in verbis:

$\S 8^{\circ}$ Poderá ser instituída "conta mínima", baseada em quantidade mínima de
consumo ou utilização do serviço, mediante critérios e requisitos
fundamentados em razões de segurança sanitária das pessoas e dos
ambientes em que residam ou trabalhem ou "tarifa básica" baseada em custo
mínimo fixo necessário para amortização, operação e manutenção do sistema
disponibilizado, cujas regras devem ser aprovadas pela entidade reguladora
e fiscalizadora. (BRASIL, 2004)

De acordo com a resolução normativa $N^{\circ}$ 0125/2018, da Agencia Goiana de Regulamentação - AGR (2018), a estrutura tarifária será composta por duas espécies de cobranças: a tarifação básica fixa, que é classificada de acordo com a categoria do estabelecimento estudado e sujeito a reajustes anuais conforme a necessidade, e a tarifa diretamente associada ao consumo mensal de água, que corresponde à água, a coleta e o tratamento e ao tratamento de esgoto.

Portanto, independentemente do consumo, sempre haverá uma tarifa básica fixa préestabelecida e passiva de ajustes, como pode ser vista na Tabela 1 (valores validos para a tarifação a partir do mês de abril de 2018). Nota-se que tal tarifa varia apenas em função da categoria da economia consumidora.

TABELA 1 - Tarifas Básicas - SANEAGO

\begin{tabular}{cc} 
Categoria & Tarifa $(\mathbf{R} \mathbf{\text { mês }})$ \\
\hline \hline Residencial Social & 6,36 \\
\hline Residencial Normal & 12,71 \\
\hline Comercial I & 12,71 \\
\hline Comercial II & 6,36 \\
\hline Industrial & 12,71 \\
\hline Pública & 12,71 \\
\hline \multicolumn{2}{c}{ FONTE: Adaptado de AGR (2018) }
\end{tabular}

A tarifa básica é realizada, segundo a concessionária, para garantir a viabilidade econômica da instituição frente às possíveis reformas, manutenções, pagamento de 
dívidas e investimentos do sistema de distribuição de água, coleta e tratamento de esgoto. O faturamento apurado pela SANEAGO é subdividido em quatro serviços: o custo mínimo fixo, a tarifa de consumo de água conforme a categoria do imóvel, a coleta/afastamento e o tratamento de esgoto. Para fins comparativos, foi feita uma adaptação, no qual a tarifa de coleta/afastamento e tratamento de esgoto é representada como um único serviço associado.

Evidentemente, ainda existe a tarifa por consumo efetivo baseado na mesma estrutura de blocos, só que, no caso da SANEAGO, a primeira faixa ainda é custeada com base no $\mathrm{m}^{3}$ distribuído.

Compilando as informações da estrutura tarifária da SANEAGO referente ao consumo residencial normal observa-se a Tabela 2.

TABELA 2 - Resumo da estrutura tarifária da SANEAGO referente ao consumo residencial normal

\begin{tabular}{cccc}
\hline $\begin{array}{c}\text { Faixa de } \\
\text { consumo } \\
\left(\mathbf{m}^{\mathbf{3}}\right)\end{array}$ & Limite & $\begin{array}{c}\text { Consumo } \\
\left(\mathbf{m}^{3}\right)\end{array}$ & Consumo total (R\$) \\
\hline \hline \multirow{2}{*}{$\mathbf{0 - 1 0}$} & Inferior & $\mathbf{0}$ & 12,71 \\
& $*$ & $\mathbf{1}$ & 21,11 \\
& Superior & $\mathbf{1 0}$ & 96,71 \\
\hline \multirow{2}{*}{$\mathbf{1 1 - 1 5}$} & Inferior & $\mathbf{1 1}$ & 117,21 \\
& Superior & $\mathbf{1 5}$ & 155,21 \\
\hline \multirow{2}{*}{$\mathbf{1 6 - 2 0}$} & Inferior & $\mathbf{1 6}$ & 186,47 \\
& Superior & $\mathbf{2 0}$ & 229,91 \\
\hline \multirow{2}{*}{$\mathbf{2 1 - 2 5}$} & Inferior & $\mathbf{2 1}$ & 271,43 \\
& Superior & $\mathbf{2 5}$ & 320,71 \\
\hline \multirow{2}{*}{$\mathbf{2 6 - 3 0}$} & Inferior & $\mathbf{2 6}$ & 374,63 \\
& Superior & $\mathbf{3 0}$ & 430,31 \\
\hline \multirow{2}{*}{$\mathbf{3 1 - 4 0}$} & Inferior & $\mathbf{3 1}$ & 504,99 \\
& Superior & $\mathbf{4 0}$ & 647,91 \\
\hline \multirow{2}{*}{$\mathbf{4 1 - 5 0}$} & Inferior & $\mathbf{4 1}$ & 749,07 \\
& Superior & $\mathbf{5 0}$ & 910,71 \\
\hline \hline $\mathbf{+ 5 0}$ & inferior & $\mathbf{5 1}$ & 1057,19 \\
\hline & & & FONTE: Adaptado de AGR (2018) \\
\hline
\end{tabular}

\section{METODOLOGIA}

Para a realização do estudo inicialmente levantou-se o consumo de água de diferentes perfis de agentes consumidores a fim de definir um valor referência de consumo mensal médio de diferentes edifícios residenciais, situados na cidade de Goiânia, considerandose as suas unidades consumidoras associadas à quantidade de habitantes. Por meio deste valor referência foram criados diferentes cenários de consumo alterando-se a porcentagem de agentes consumidores por unidade habitacional (Tabela 3). 
TABELA 3 - Cenários de estudo

\begin{tabular}{ccccccc}
\hline $\begin{array}{c}\mathbf{N}^{\circ} \text { de habitantes por } \\
\text { unidade } \\
\text { habitacional }\end{array}$ & Cenário 1 & Cenário 2 & Cenário 3 & Cenário 4 & Cenário 5 & Cenário 6 \\
\hline \hline Desocupado & $10 \%$ & $15 \%$ & $25 \%$ & $35 \%$ & $10 \%$ & $5 \%$ \\
\hline 1 & $15 \%$ & $25 \%$ & $35 \%$ & $10 \%$ & $5 \%$ & $10 \%$ \\
\hline 2 & $25 \%$ & $35 \%$ & $10 \%$ & $5 \%$ & $10 \%$ & $15 \%$ \\
\hline 3 & $35 \%$ & $10 \%$ & $5 \%$ & $10 \%$ & $15 \%$ & $25 \%$ \\
\hline 4 & $10 \%$ & $5 \%$ & $10 \%$ & $15 \%$ & $25 \%$ & $35 \%$ \\
\hline 5 & $5 \%$ & $10 \%$ & $15 \%$ & $25 \%$ & $35 \%$ & $10 \%$ \\
\hline \hline
\end{tabular}

O edifício hipotético utilizado para os cálculos é composto por 20 pavimentos, sendo 4 apartamentos por andar, porém vale ressaltar que a quantidade total de apartamentos não influencia diretamente nas análises, uma vez que se utilizou taxas de ocupação em porcentagem e dados de consumo referentes à diferentes números de habitantes por unidade. É evidente que os cenários concebidos só serão passíveis de existir a partir de um número mínimo de apartamentos e, para tanto, optou-se por essa conformação.

\section{RESULTADOS}

\subsection{Consumo médio}

Segue, na Tabela 4, a média final dos dados de consumo obtido para cada relação habitante por unidade analisada.

TABELA 4 - Consumo médio até 5 moradores por unidade

\begin{tabular}{ccc}
\hline Quantidade de moradores & Medições analisadas & Consumo médio $\left(\mathbf{m}^{\mathbf{3}} / \mathbf{m e ̂ s}\right)$ \\
\hline \hline 1 & 21 & 4,81 \\
\hline 2 & 21 & 8,24 \\
\hline 3 & 42 & 11,25 \\
\hline 4 & 77 & 15,40 \\
\hline 5 & 2 & 17,63 \\
\hline \hline & & FONTE: Autoria Própria
\end{tabular}

\section{2 Ánalise dos cenários}

A partir do consumo médio obtido, apresenta-se, na Tabela 5, as tarifas simuladas em cada concessionária com SMI. 
TABELA 5 - Tarifas SMI simuladas nas concessionárias

\begin{tabular}{ccc}
\hline hab/unid & Consumo $\left(\mathbf{m}^{3}\right)$ & \begin{tabular}{c} 
Tarifa - SMI (R\$) \\
\hline
\end{tabular} \\
\hline \hline $\mathbf{1}$ & 4,81 & SANEAGO \\
\hline $\mathbf{2}$ & 8,24 & 53,11 \\
\hline $\mathbf{3}$ & 11,25 & 81,91 \\
\hline $\mathbf{4}$ & 15,40 & 119,62 \\
\hline $\mathbf{5}$ & 17,63 & 179,98 \\
\hline \hline & & FONTE: Autoria Própria
\end{tabular}

Apoiado nos dados de consumo obtidos e nos cenários estipulados, foi possível calcularse o custo total para cada apartamento considerando-se um SMC, apresentados na Tabela 6. Salienta-se que os custos encontrados para o caso coletivo foram aplicados apenas às unidades consumidoras habitadas com o fornecimento de água ligado.

TABELA 6- Tarifas no Edifício Hipotético para os cenários - SMC

\begin{tabular}{cccc}
\hline Cenário & $\begin{array}{c}\text { Consumo Total } \\
\left(\mathbf{m}^{\mathbf{3}}\right)\end{array}$ & $\begin{array}{c}\text { Média por unidade } \\
\text { habitada }\left(\mathbf{m}^{\mathbf{3}}\right)\end{array}$ & Tarifa rateada (R\$) \\
\hline \hline $\mathbf{1}$ & 731,32 & 10,16 & SANEAGO \\
\hline $\mathbf{2}$ & 619,52 & 9,11 & 96,65 \\
\hline $\mathbf{3}$ & 580,35 & 9,67 & 76,69 \\
\hline $\mathbf{4}$ & 698,85 & 13,44 & 81,41 \\
\hline $\mathbf{5}$ & 1021,83 & 14,19 & 127,83 \\
\hline $\mathbf{6}$ & 934,71 & 12,30 & 134,98 \\
\hline \hline & & & FONTE: Autoria Própria
\end{tabular}

De posse dos custos totais para cada cenário SMC e SMI respectivos, confrontou-se tais valores e determinou-se uma quantidade inteira mínima de habitantes por unidade consumidora a partir da qual o SMC poderia se tornar economicamente mais viável para o consumidor do que o SMI. Consequentemente, via as taxas de ocupação estabelecidas em cada situação, determinou-se a porcentagem de apartamentos que seriam favorecidos pelo SMC. A Tabela 7 apresenta as porcentagens de beneficiados (valores em azul $\downarrow$ ) e desfavorecidos (valores em vermelho $\uparrow$ ) e sua respectiva porcentagem de apartamentos.

TABELA 7 - Avaliação dos cenários favorecidos pelo SMC

\begin{tabular}{ccccccccccccc}
\hline & \multicolumn{3}{c}{ Cenário 1 } & \multicolumn{2}{c}{ Cenário 2 } & \multicolumn{2}{c}{ Cenário 3 } & \multicolumn{2}{c}{ Cenário 4 } & \multicolumn{2}{c}{ Cenário 5 } & \multicolumn{2}{c}{ Cenário 6 } \\
\hline \hline hab/unid & Econ. & Ap. & Econ. & Ap. & Econ. & Ap. & Econ. & Ap. & Econ. & Ap. & Econ. & Ap. \\
\hline $\mathbf{1}$ & $\uparrow 82 \%$ & $15 \%$ & $\uparrow 44 \%$ & $25 \%$ & $\uparrow 53 \%$ & $35 \%$ & $\uparrow 141 \%$ & $10 \%$ & $\uparrow 154 \%$ & $5 \%$ & $\uparrow 120 \%$ & $10 \%$ \\
\hline $\mathbf{2}$ & $\uparrow 18 \%$ & $25 \%$ & $\downarrow 6 \%$ & $35 \%$ & $\downarrow 1 \%$ & $10 \%$ & $\uparrow 56 \%$ & $5 \%$ & $\uparrow 65 \%$ & $10 \%$ & $\uparrow 43 \%$ & $15 \%$ \\
\hline $\mathbf{3}$ & $\downarrow 19 \%$ & $35 \%$ & $\downarrow 36 \%$ & $10 \%$ & $\downarrow 32 \%$ & $5 \%$ & $\uparrow 7 \%$ & $10 \%$ & $\uparrow 13 \%$ & $15 \%$ & $\downarrow 2 \%$ & $25 \%$ \\
\hline $\mathbf{4}$ & $\downarrow 46 \%$ & $10 \%$ & $\downarrow 57 \%$ & $5 \%$ & $\downarrow 55 \%$ & $10 \%$ & $\downarrow 29 \%$ & $15 \%$ & $\downarrow 25 \%$ & $25 \%$ & $\downarrow 35 \%$ & $35 \%$ \\
\hline $\mathbf{5}$ & $\downarrow 53 \%$ & $5 \%$ & $\downarrow 62 \%$ & $10 \%$ & $\downarrow 60 \%$ & $15 \%$ & $\downarrow 37 \%$ & $25 \%$ & $\downarrow 34 \%$ & $35 \%$ & $\downarrow 43 \%$ & $10 \%$ \\
\hline \hline
\end{tabular}




\section{CONCLUSÕES OU CONSIDERAÇÕES FINAIS}

Um cenário ideal para a adoção do SMC seria o de um edifício totalmente habitado com a mesma quantidade de moradores por apartamento, que permitisse reduzir a tarifa básica fixa de múltiplas moradias para uma única, incidida apenas no hidrômetro geral, acarretando uma economia para todos os apartamentos de aproximadamente igual à tarifa mínima.

A partir do estudo realizado, conclui-se que a tarifa básica possui um peso grande em relação à tarifa mensal de água para as unidades habitacionais com poucos moradores, uma vez que a tarifa representa cerca de $31,5 \%$ do valor médio pago por unidades com 1 habitante e $18,4 \%$ acima do valor pago para unidades com 2 habitantes.

Apesar da proporção da tarifa básica representar uma parcela significativa da tarifação incidida em apartamentos com até dois habitantes, o SMI, ainda sim, resulta em uma redução global de consumo de água e, consequentemente, em um valor menor de cobrança devido a essa economia.

\section{REFERENCIAS}

AGR - AGÊNCIA GOIANA DE REGULAÇÃO, CONTROLE E FISCALIZAÇÃO DE SERVIÇOS PÚBLICOS. Resolução da diretoria ${ }^{\circ}$ 071/2018. 2018. Disponível em: <https://www.saneago.com.br/site/agencia/tabela_tarifas_agua_esgoto.pdf $>$. Acesso em: 10 de Setembro de 2018.

AGÊNCIA NACIONAL DE ÁGUA (ANA) - Ministério do Meio Ambiente. Conservação e reuso da água em edificações. São Paulo, 2005. 152 p.

BRASIL, Lei $n^{\circ}$ 13.312, de 12 de julho de 2016. Altera a Lei $n=11.445$, de 5 de janeiro de 2007, que estabelece diretrizes nacionais para o saneamento básico, para tornar obrigatória a medição individualizada do consumo hídrico nas novas edificações condominiais. Disponível em: <http://www.planalto.gov.br/ccivil_03/LEIS/L9433.htm>. Acesso em: 19 de setembro de 2018.

BRASIL, Lei no 14.939, de 15 de setembro de 2004. Institui o Marco Regulatório da Prestação de Serviços de Abastecimento de Água e Esgotamento Sanitário, cria o Conselho Estadual de Saneamento - CESAM e dá outras providências. Disponível em: <http://www.gabinetecivil.goias.gov.br/leis_ordinarias/2004/lei_14939.htm>. Acesso em 31/10/2018.

CARVALHAES, M. DA C. Gestão da água em edifícios habitacionais de múltiplos pavimentos: Análise da Operação em Sistemas de Medição Individualizada em São Paulo entre 1980 e 2015, 2016. Dissertação apresentada à Universidade Presbiteriana Mackenzie como requisito parcial à obtenção de título de Mestre em Arquitetura e Urbanismo.

CARVALHO JR., W. SILVEIRA, A. Sistema de medição individualizado de água-Análise de viabilidade de implantação, Estudo de Caso: Edifico Residencial New York, Cuiabá/ MT. In: 4 o Encontro de Engenharia e Tecnologia dos Campos Gerais, 2008, Campos Gerais, Brasil. 7 p.

CHAIB, E. B A. Avaliação de estratégias para a redução do consumo de água de abastecimento como meio de diminuição da pressão sobre os recursos hídricos. 2016, 254 p. Tese de Doutorado. Escola de Engenharia da UFMG, Belo Horizonte, 2016.

GUEDES, E.S.S. JÚNIOR, G.B.A. Comparativo de custos da medição individual e coletiva de água fria em edifícios residenciais. In: VI Congresso Brasileiro de Gestão Ambiental, 2015, Porto Alegre, Brasil. 7 p.

PERES, A. R. B. Avaliação Durante Operação de Sistemas de medição Individualizada de Água em Edifícios Residenciais, 2006. Dissertação apresentada no Curso de Mestrado em Engenharia Civil da Universidade Federal de Goiás para a obtenção do título de Mestre em Engenharia Civil. 
PERUCH, D.H. BACK, N. Comparativo do Consumo de Água em Edificações Verticais Residenciais Multifamiliares com Hidrômetros Individuais e Coletivos. 2013. Artigo submetido à UNESC Universidade do Extremo Sul Catarinense, 2013.

ROZAS, N. PRADO, R.T.A. Implantação de sistemas de leitura automática de medidores de insumos prediais. 2002. 21 f. Dissertação (Mestrado em Engenharia Civil) - Departamento de Engenharia de Construção Civil, Universidade de São Paulo, São Paulo, 2002.

YAMADA, E. S.; PRADO, R. T. A.; IOSHIMOTO, E. Os Impactos da medição individualizada do consumo de água em edifícios residenciais multifamiliares. 2001. 125 p. Dissertação (Mestrado). Escola Politécnica da Universidade de São Paulo, São Paulo, 2001 